\title{
Identification of removal parameters at combined grinding of conductive ceramic materials
}

\author{
Sergey Bratan ${ }^{1, *}$, Stanislav Roshchupkin ${ }^{1}$, Alexandr Kolesov ${ }^{1}$, and Boris Bogutsky ${ }^{1}$ \\ ${ }^{1}$ Sevastopol State University,33, Universitetskaya St., Sevastopol, 299053, Russia
}

\begin{abstract}
The models of combined grinding for processing of conductive ceramic materials which allows to calculate the material removal and to optimize the machining process on the automated equipment were obtain. The models take into account that process has a stochastic nature and that material is removed by a combination of microcutting-chipping, brittle volumetric fracture and erosive material removal.
\end{abstract}

At the finishing of conductive brittle materials, for example, specialized ceramics and conductive ceramic coatings, combined grinding methods are used, in which the material is removed by a combination of microcutting-chipping, brittle volumetric fracture and erosive material removal.

Automation of such processes in modern conditions is possible in the presence of rational operation control cycles. Their development by empirical means does not allow to widely implement the processes of combined grinding due to the large volume of preliminary studies.

Creating a mathematical model of the machining process makes it possible to automate the design of the finish operation and optimize it according to the required criterion.

To obtain a mathematical model that allows to calculate the removal of material in the presence of microcutting-chipping, brittle volumetric fracture and erosive material removal, the process of interaction of the tool with the workpiece at the micro level is considered. Diamond grains are randomly distributed in the volume of the grinding wheel, so the process of their interaction with the material being processed is random $[1,2]$.

The removal of material as a result of erosion and abrasive work is a random process, which can be characterized by a complete probability of material removal. For any point of the workpiece surface, one can consider a random event consisting in the joint failure of this point by erosion, microcutting-chipping and brittle volume destruction, then the total probability of removal is determined by:

$$
P(\bar{M})=P_{1}(\bar{M}) \cdot P_{2}(\bar{M}) \cdot P_{3}(\bar{M}),
$$

where $P_{1}(\bar{M})$ - is the probability that the material has not been removed due to micro-cutting; $P_{2}(\bar{M})$ - the probability that the material is not removed due to volumetric brittle fracture, $P_{3}(\bar{M})$ - the probability of erosion of the material. The probability of material failure at a time due to microcutting is determined by the dependence [2]

$$
P_{1}(\bar{M})=\exp \left[-a_{0}-\int_{t_{o}}^{t} a_{1 i}(y, \tau) d \tau\right]
$$

where $y$ - distance from the outer surface of the workpiece to the considered level. The indicator $a_{1}$ for each pass is given by

$$
\Delta a_{1}(y, \tau)=\kappa_{c} b_{3}(y, \tau) \Delta \lambda
$$

where $k_{c}$ - chip formation ratio; $b_{3}(y)$ - grain width at the level $y ; \lambda \Delta$ - the number of diamond grains that have passed through a unit cross section.

For conditions where brittle materials (various types of ceramics) are ground, $k_{c}=1$. In the process of grinding, a certain number of grains perform a microcutting of the surface of the workpiece, and the other part of the grains immediately cleaves the material. Therefore, when calculating the indicator $a_{1}$ it is necessary to take into account only those grains that can cut the material, i.e. from the total number of grains it is necessary to subtract the grains that produce the spalling.

$$
\Delta a_{1}(y, \tau)=b_{3}(y, \tau) \Delta \lambda\left(1-P_{c \kappa}\right),
$$

where $P_{c k}$ - is the probability of volumetric brittle fracture of the workpiece material when interacting with the grain of the tool.

When the grain profile is approximated by a power law (in the simplest case, a paraboloid of revolution), we obtain

\footnotetext{
* Corresponding author: bratan@sevsu.ru
} 


$$
b_{3}(y, \tau)=C_{b}\left(t_{\phi}-y-u\right)^{m},
$$

where $C_{b}, m$ - are the coefficients of the grain shape; $t_{\phi}$ the actual depth of microcutting; $u$ - the distance from the conditional outer surface of the tool to the top of the grain.

Through a unit section $1 \cdot \Delta \mathrm{u}$ in time $\Delta \tau$ passes the $\Delta \lambda$ grain of the tool

$$
\Delta \lambda=V_{\kappa} n_{3} f(u) d u d \tau,
$$

where $n_{3}$ - the number of grains per unit volume of the working layer of the tool; $V_{k}$ - tool feed speed.

In turn, the distribution function of the number of working grains of the tool along the coordinate can be approximated by the dependence [2]

$$
f(u)=\frac{\chi}{H_{u}^{\chi}} u^{\chi-1}
$$

where $H_{u}$ the value of the layer of the working surface of the circle within which it is counted $n_{3}$.

After substituting (5) and (6) in (4) and integrating with respect to $u$ and $\tau$, it follows that (6):

$$
a_{1}(y, \tau)=n_{3} V_{\kappa} \kappa_{c} \int_{t_{0}}^{t_{t}} \int_{0}^{-y} b_{3} f(u)\left(1-P_{c \kappa}\right) d u d \tau
$$

The probability of spalling $P_{c k}$ is determined by the mode of grinding and the location of the grain in the volume of the tool. For its calculation can be used. the dependences of A. Korolyov [1], obtained by analyzing the process of dressing the abrasive tool and allowing to determine the probability of volumetric destruction of the grinding wheel grains. The process of dressing abrasive wheels in a certain sense is similar to the process of diamond grinding. However, these dependencies are complex for practical use. Therefore, it is desirable to replace them with approximations so that an analytic calculation of the integral (6) becomes possible. In this capacity, the dependence

$$
P_{c \kappa}=P_{0}\left[1-\left(\frac{u}{t_{\phi}}\right)^{\beta}\right] .
$$

For the level $u=0$ и $P_{c k}=P_{0}$, where $P_{0}$ it is determined by grinding modes and tool characteristics. For the level $u=t_{\phi}$ (the outer surface of the workpiece) $P_{c k}=0$. The coefficients $P_{0}$ and for specific grinding conditions can be calculated from the dependences [1]. for specific grinding conditions can be calculated from the dependences [1]. For this, it is sufficient to calculate only two values $P_{c k}$ and $u$. Substituting (7) into (6), we obtain

$$
\begin{aligned}
& a_{1}(y, \tau)=n_{3} V_{\kappa} \kappa_{c} \cdot \\
& \cdot \int_{t_{0}}^{t_{\phi}} \int_{0}^{-y} C_{b}\left(t_{\phi}-y-u\right)^{m} \frac{\chi}{H_{u}^{\chi}} u^{\chi-1}\left[1-P_{0}+P_{0}\left(\frac{u}{t_{\phi}}\right)^{\beta}\right] d u d \tau
\end{aligned}
$$

The integral (8) is calculated for $\tau=t_{0}$ :

$a_{1}(y, \tau)=n_{3} V_{\kappa} \kappa_{c} \cdot \int_{t_{0}}^{t} \int_{0}^{t_{\phi}-y} \frac{C_{b}\left(t_{\phi}-y-u\right)^{m} \chi u^{\chi-1}}{H_{u}^{\chi}}$.

$\cdot\left(1-P_{0}\right) d u d \tau++n_{3} V_{\kappa} \kappa_{c} \int_{t_{0}}^{t} \int_{0}^{t_{\phi}-y} \frac{C_{b}\left(t_{\phi}-y-u\right) \chi u^{\chi-1}}{H_{u}^{\chi}}$.

$\cdot P_{0}\left(\frac{u}{t_{\phi}}\right)^{\beta} d u d \tau=$

$=\frac{C_{b} n_{3} V_{\kappa} \kappa_{c} \Gamma(m+1) \Gamma(\chi)\left(t-t_{0}\right)\left(t_{\phi}-t_{0}\right)^{m+\chi}}{H_{u}{ }^{\chi} \Gamma(m+\chi+1)(m+\chi+1)}$.

$\cdot\left(1-P_{0}\right)+C_{b} n_{3} V_{\kappa} \kappa_{c} \Gamma(m+1) \Gamma(\chi+\beta) P_{0}$.

$\cdot \frac{\left(t-t_{0}\right)\left(t_{\phi}-t_{0}\right)^{m+\chi+\beta}}{t_{\phi}^{\beta} H_{u}^{\chi} \Gamma(m+\chi+\beta)(m+\chi+\beta+1)}$.

Taking into account the notation:

$$
\begin{aligned}
A= & \frac{\Gamma(m+1) \Gamma(\chi)}{\Gamma(m+\chi+1)(m+\chi+1)}\left(1-P_{0}\right) ; \\
B= & \frac{\Gamma(m+1) \Gamma(\chi+\beta)}{\Gamma(m+\chi+\beta+1)(m+\chi+\beta+1)} P_{0} ; \\
C= & \frac{C_{b} n_{3} V_{\kappa} \kappa_{c}}{H_{u}{ }^{\chi}} ; \text { (9) takes the form } \\
& a(y, \tau)=C A\left(t-t_{0}\right)\left(t_{\phi}-y\right)^{m+\chi}\left(1-P_{0}\right)+ \\
& +\frac{C B}{t_{\phi}{ }^{\beta}}\left(t-t_{0}\right)\left(t_{\phi}-y\right)^{m+\chi+\beta} P_{0}= \\
& =C\left(t-t_{0}\right)\left(t_{\phi}-y\right)^{m+\chi}\left[A+\frac{B}{t_{\phi}{ }^{\beta}}\left(t_{\phi}-y\right)^{\beta}\right] .
\end{aligned}
$$

After each time interval $\Delta \tau$, the value $y$ changes by the amount of removal $\Delta h$

$$
\begin{aligned}
& y\left(\tau_{1}\right)=y+\Delta h \\
& y\left(\tau_{2}\right)=y+2 \Delta h \\
& y\left(\tau_{3}\right)=y+3 \Delta h
\end{aligned}
$$

Hence, taking (2) into account, we obtain (11) 


$$
P_{1}(\bar{M})=\exp \left(\begin{array}{l}
-\int_{t_{0}}^{t} C\left(t-t_{0}\right)\left(t_{\phi}-y-\tau \Delta h\right)^{m+\chi} . \\
\left.A+\frac{B}{t_{\phi} \beta}\left(t_{\phi}-y-\tau \Delta h\right)^{\beta}\right) d \tau
\end{array}\right),
$$

where $\tau$ - is the current time.

After integrating over $\tau$, expression (11) takes the form (12):

$$
\begin{aligned}
& P_{1}(\bar{M})=\exp \left(-G_{1} \frac{\left(t_{\phi}-y-t \Delta h\right)^{m_{1}}-\left(t_{\phi}-y-t_{0} \Delta h\right)^{m_{1}}}{\left(t_{\phi}-y\right) m_{1}}+\right. \\
& \left.+L_{1} \frac{\left(t_{\phi}-y-t \Delta h\right)^{m_{2}}-\left(t_{\phi}-y-t_{0} \Delta h\right)^{m_{2}}}{\left(t_{\phi}-y\right) m_{2}}\right),
\end{aligned}
$$

$$
\text { where } \quad G_{1}=C A ; L_{1}=C B, \quad m_{1}=m+\chi+1 \text {, }
$$
$m_{2}=m+\chi+\beta+1$.

The probability of not removing material due to volumetric brittle fracture will be written as

$$
P_{2}(\bar{M})=\exp \left(-a_{0}-\int_{t_{0}}^{t} a_{2}(y, \tau) d \tau\right) .
$$

For the case under consideration, the width of a single risk $b_{x}$, as a result of the interaction between the grain of the instrument and the ceramic part, is greater than its actual width $b_{3}$.

For the approximation $b_{x}$, we use the power-law dependence

$$
b_{x}(y, \tau)=C_{b x}\left(t_{\phi}-y-u+\Delta h_{x}\right)^{m_{x}},
$$

where $\Delta h_{x}$ - is the value of the increment of material removal during the brittle fracture of the ceramic.

In this case, the exponent $\mathrm{a}_{2}$ is equal to

$$
\Delta a_{2}(y, \tau)=b_{x}(y, \tau) \Delta \lambda P_{c \kappa} .
$$

Transformations similar to the derivation of dependencies for $a_{1}(y)$, will lead to the dependence

$$
P_{2}(\bar{M})=\exp \left[-a_{0}-\int_{t_{0}}^{t} \cdot\left(A_{2}+\frac{B}{t_{\phi}^{\beta}}\left(t_{\phi}+\Delta h_{x}-y-\tau \Delta h\right)^{\beta}\right) d \tau\right]
$$

$$
\text { Where } A_{2}=\frac{\Gamma(m+1) \Gamma(\chi)}{\Gamma(m+\chi+1)(m+\chi+1)} P_{0} .
$$

After integrating over $\tau$, expression (13) takes the form

$$
\begin{aligned}
& P_{2}(\bar{M})=\exp \left(-G_{2} \frac{\left(t_{\phi}-y+\Delta h_{x}-t \Delta h\right)^{m_{1}}}{\left(t_{\phi}-y\right) m_{1}}-\right. \\
& -\frac{\left(t_{\phi}-y+\Delta h_{x}-t_{0} \Delta h\right)^{m_{1}}}{\left(t_{\phi}-y\right) m_{1}}+ \\
& \left.+L_{1} \frac{\left(t_{\phi}-y+\Delta h_{x}-t \Delta h\right)^{m_{2}}-\left(t_{\phi}-y+\Delta h_{x}-t_{0} \Delta h\right)^{m_{2}}}{t_{\phi}^{\beta}\left(t_{\phi}-y\right) m_{2}}\right),
\end{aligned}
$$

where $G_{2}=C A_{2}$.

To describe the probability of erosion failure, let us consider the processes taking place in the presence of erosion discharges in the processing zone.

Traces from erosion discharges can form on the surface of the billet (Fig. 1).

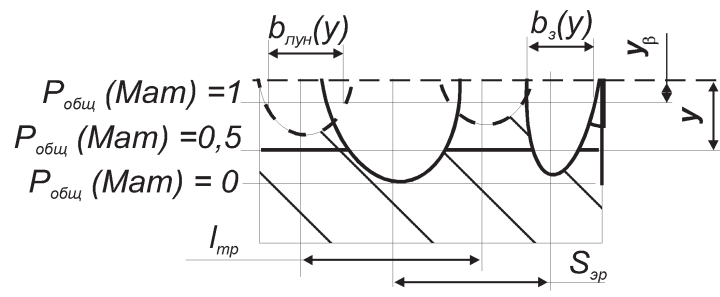

Fig.1. Calculation scheme

If two adjacent wells overlap, the material between them will be completely removed. This condition for any level $y$ can be written:

$$
0<S_{\text {эр }} \leq 0.5\left[b_{\text {эр } 1}(y)+b_{\text {эр2 }}(y)\right],
$$

Where $S_{\supset p}$ - is the distance between two adjacent holes on the surface in the section under consideration, $b_{\text {эр } 1}(y), b_{\text {эр } 2}(y)$ - is the width of two neighboring lunar holes at the level $\mathrm{y}$.

If the width of the holes at the level $y$ is equal, then the material removal condition can be rewritten as follows: $0<S_{\text {эр }} \leq b_{\text {эр }}(y)$.

Obviously, the probability of erosive removal of the workpiece $P_{3}(y)$ at the level y will be equal to the probability that the random variable Sэp falls on the segment from 0 to $b_{\text {эр }}(y)$. If the density of the probability distribution of a continuous quantity $S_{э p}$ is denoted by $f(S \ni p)$, then the probability of removing the material $P_{3}(y)$ :

$$
P_{3}(y)=P\left(0<S_{\ni p} \leq b_{\ni p}(y)\right)=\int_{0}^{b_{\text {эp }}(y)} f\left(S_{\ni p}\right) d S_{\ni p}
$$

Therefore, the appearance of holes on the surface of the workpiece with a distance Sэp after passing through a single section of the circle can be regarded as a stationary ordinary flow with a limited aftereffect. Since a large number of single tool surfaces pass through each section of the workpiece, the stream of events Sэp in the section under consideration is the sum of a large number of stationary, ordinary flows with a limited aftereffect. 
From the theory of queuing it follows that the flow Sэp can be assumed arbitrarily close to the simplest one, and the distribution density

$$
f\left(S_{\ni p}\right)=\lambda \cdot e^{-\lambda S_{\ni p}}
$$

Then we get an expression for the probability of material removal:

$$
P_{3}(y)=\int_{0}^{b_{э p}(y)} \lambda_{л y H} \cdot e^{-\lambda_{\pi y h} S_{э p}} d S_{\ni p}=1-e^{-\lambda b_{э p}(y)}=1-e^{-a_{3}(y)}
$$

where $\lambda_{\text {лун }}$ - the average number of holes per unit length of the section of the workpiece.

Thus, the probability of erosion of the material at any of the considered levels $y$ is determined by the width of the generating holes and their number per unit length of the cross section.

$$
a_{3}(y)=\lambda_{\text {лун }} b_{\text {эр }}(y) .
$$

The shape of the hole is usually modeled by a spherical segment with a diameter at the surface $-d_{\pi}$ and depth - $h_{\pi}$ [3]. The surface on which the holes are formed is constantly shifted deep into the workpiece, because the workpiece material is removed. The width of the well bзp at the level $y-y_{\beta}$ from the surface of the occurrence of the discharge (Fig. 1) is determined from the geometric analysis of the spherical segment:

$$
\left.\begin{array}{lrl}
b_{э р}(y)=0, & \text { at } y_{\beta}>y>y_{\beta}+h_{л} \\
b_{э р}(y)=R \sqrt{h_{л}-y_{э р},} & y_{\beta} \leq y \leq y_{\beta}+h_{л}
\end{array}\right\},
$$

where $R$ - is the index that determines the diameter of the well.

Since erosion discharges occur at the places where chipped particles of the material (shavings) are formed [3], and the share of random discharges in the total volume is negligible and can be neglected, the number of emerging holes depends directly on the number of cutting grains and on the structure of the tool.

To determine the probability of erosion destruction of the workpiece $\Delta z$ when grinding the periphery of the circle, consider the section of the part in the contact zone.

During the time $\Delta \tau$ the section of the workpiece is displaced by a distance and through it passes a section of the circle with the length of the arc, $\left(V_{\kappa} \pm V_{u}\right) \Delta \tau$, where $V_{\kappa}$ - is the circumferential speed of the circle, $V_{u}$ - the speed of the workpiece. Of the total number of cutting elements on the surface of the tool, chips can form grains whose vertices are located in the layer of the circle in height $u \leq t_{\phi}-y_{\beta}$, where $t_{\phi}$ - is the actual depth of cutting.

The number of chips $\Delta \lambda$ formed by the elementary layer of the tool is calculated from the grain distribution density $f_{3}(u)(5)$, the generated chips create a high local electric field strength and thereby cause the erosion discharge. However, studies show that not all chips can cause breakdown of the interelectrode gap. This is due to the fact that there is a pause between two digits at one point in space $\tau_{n}$ for the duration of the deionization of the gap [3]. The length of the necessary pause is determined by the medium filling the MEP.

Thus, the number of discharges occurring in the contact zone from the action of the elementary surface of the instrument, we determine:

$$
\Delta N_{\ni p}=K_{\ni p} \Delta \lambda
$$

where $K_{\ni p}$ - is a coefficient that takes into account the number of chips that do not produce a discharge.

Change the parameter $a_{3}(y, \tau)$ as the increment of the sum of the transverse dimensions of the holes (18) and calculate:

$$
\begin{aligned}
& \Delta a_{3}(y, \tau)=k_{c} b_{э р}(y) \Delta N_{э р}= \\
& =k_{c} b_{\ni p}(y) n_{3} f_{3}(u) \Delta u\left(V_{\kappa} \pm V_{u}\right) \Delta \tau
\end{aligned} .
$$

Replacing $\tau$ by $\frac{z}{V_{u}}$ calculate the value of the indicator $a_{\ni p}(y, \tau)$ at the point $z$ of contact zone.

$$
\begin{aligned}
& a_{3}(y, \tau)=R k_{c} \frac{\left(V_{\kappa} \pm V_{u}\right)}{V_{u}} n_{3} . \\
& \cdot \int_{0}^{t_{\phi}-y_{\beta}} \int_{-L}^{z} f_{3}(u) \sqrt{\left(h_{\pi}-y+y_{\beta}\right)} d u d z
\end{aligned}
$$

where $L$ - half the length of the contact zone, $z$ - the coordinate along the length of the contact zone.

Expression (22) represents a generalized dependence for determining the probability of erosive removal of the workpiece material.

Substituting values $h_{3}$ for the depth of the erosion hole respect to the height $f_{3}(u)$, we will be able to calculate the total probability of removing the material $P_{\Sigma}(y, z)$ sequentially at all levels of the workpiece:

$$
\begin{aligned}
& P_{\Sigma}(y, z)=1-\bar{P}_{1}(y, z) \bar{P}_{2}(y, z) \bar{P}_{3}(y, z)= \\
& =1-\exp \left[-a_{0}(y, z)-a_{1}(y, z)-a_{2}(y, z)-a_{3}(y, z)\right]
\end{aligned}
$$

The common layer of the removed material $y_{\beta}$ can be formally written:

$$
y_{\beta}=\int_{0}^{t_{\phi}} 1\left(P_{\Sigma}(y, z)-\beta_{M}\right) d y,
$$

where $1\left(P_{\Sigma}(y, z)-\beta_{M}\right)-$ is the unit function $P_{\Sigma}(y, z)$, which when value $\beta_{M}$ takes a value of one; $\beta_{M}$ - the accepted confidence level is usually 0.95 .

There was considered an approach to modeling of the combined grinding process allows to optimize the 
processing and shorten the preparation time of effective processing cycles on automated equipment.

\section{References}

1. A.V. Korolyov, Investigation of tool and workpiece shaping at abrasive cutting (Saratov, 1975) (in Russ.)

2. Yu.K. Novoselov, Dynamics of surface shaping in abrasive processing, (LAP LAMBERT Academic Publishing. Saarbrucken, Deutschland, P.317 2017)

3. S. Bratan, Technological bases of quality assurance and increase in stability of high-productive fine grinding, Doctoral thesis (Odessa, 2006)

4. S. Bratan, The probabilistic approach for modeling of electroerosion grinding, Bulletin of SevGTU. Series - Automation and control, Sevastopol, SevGTU Publ., Vol.7, 1997, pp. 140-144. (in Russ.)

5. I.D. Marinescu, M. Hitchiner, E. Uhlmann et al, Handbook of Machining with Grinding Wheels (CRC Press, 2007)

6. L. Yang, C. Ren, X. Jin, Experimental Study of ELID Grinding Based on the Active Control of Oxide Layer J. Mater. Process. Technol. 210(13), 1748-1753 (2010)

7. K. Fathima,M. Rahman,A. Senthil Kumar, H. S. Lim, Modeling of Ultra-Precision ELID Grinding, J. Manuf. Sci. Eng 129(2), pp. 296-302 (2006)

8. M.Z. Rahim, S. Ding J. P.T. Mo, Electrical discharge grinding of polycrystalline diamond Effect of wheel rotation, Machining Science and Technology 20(1), pp. 62-78 (2016)

9. S. K. Choudhury, V. K. Jain, M. Gupta, Electrical discharge diamond grinding of high speed steel, Machining Science and Technology 3(1), pp. 91-105 (1999) 